\title{
Cuidados odontológicos para pacientes submetidos a tratamentos antineoplásicos
}

\author{
Dental care for patients undergoing antineoplasic treatments \\ Atención para pacientes que se somenten a tratamientos antioneoplasicos
}

Recebido: 03/09/2021 | Revisado: 09/09/2021 | Aceito: 13/09/2021 | Publicado: 14/09/2021

\author{
Jéssica Karolaine Mendes Campos da Silva \\ ORCID: https://orcid.org/0000-0003-4205-6523 \\ Faculdade Patos de Minas, Brasil \\ E-mail: jessikarolaine@ hotmail.com \\ Tawane Luiza Branquinho Rios \\ ORCID: https://orcid.org/0000-0001-8348-1396 \\ Faculdade Patos de Minas, Brasil \\ E-mail: tawaneluizacp@hotmail.com \\ Cizelene do Carmo Faleiros Veloso Guedes \\ ORCID: https://orcid.org/0000-0001-7128-7537 \\ Faculdade Patos de Minas, Brasil \\ E-mail: cizelene.guedes@ faculdadepatosdeminas.edu.br
}

\begin{abstract}
Resumo
O objetivo desse trabalho é mostrar a importância do cirurgião dentista na equipe multidisciplinar e que seu papel é fundamental no acompanhamento antes, durante e após o paciente ser submetido ao tratamento antineoplásico, proporcionando cuidados preventivos e terapêuticos, com o intuito da melhora da qualidade de vida e com alívio da dor e sintomas. Metodologia: Trata-se de uma revisão de literatura com buscas bibliográficas nas bases de dados scielo e pubmed com artigos entre 1993 a 2021. Resultados: O câncer é uma proliferação desordenada das células de um tecido ou órgão que pode ser desencadeada por fatores externos e internos. O tratamento antineoplásico é divido em cirurgia, radioterapia e quimioterapia, podendo ser utilizadas de forma exclusiva ou em combinação. A radioterapia e a quimioterapia causam efeitos adversos na cavidade oral do paciente, como xerostomia, mucosite oral, trismo, disfagia, odinofagia, candidose, cárie relacionada à radiação e osteorradionecrose. É necessário que o cirurgião dentista saiba traçar o melhor protocolo de prevenção, diagnóstico e tratamento de forma individual para obter os resultados almejados. Conclusão: O cirurgião dentista exerce um papel preponderante no cuidado com pacientes submetidos a radioterapia na região de cabeça, pescoço e quimioterapia visto que estes tratamentos podem afetar a cavidade bucal e juntamente com uma equipe multidisciplinar tem a função de oferecer uma melhora na qualidade de vida do paciente e cuidados antes, durante e mesmo após o tratamento antineoplásico. É necessário que o cirurgião dentista tenha conhecimento adequado para o manejo e cuidado com os pacientes oncológicos.
\end{abstract}

Palavras-chave: Neoplasias de cabeça e pescoço; Radioterapia; Antineoplásicos; Oncologia; Cuidados odontológicos.

\begin{abstract}
Cancer is a disordered proliferation of cells in a tissue or organ that can be triggered by external and internal factors. Antineoplastic treatment is divided into surgery, radiotherapy and chemotherapy, and can be used alone or in combination. Radiotherapy and chemotherapy cause adverse effects in the patient's oral cavity, such as xerostomia, oral mucositis, trismus, dysphagia, odynophagia, candidosis, radiation-related caries and osteoradionecrosis. It is necessary for the dental surgeon to know how to design the best prevention, diagnosis and treatment protocol individually to obtain the desired results. The objective of this work is to show the importance of the dental surgeon in the multidisciplinary team and that their role is essential in monitoring before, during and after the patient is undergoing antineoplastic treatment, providing preventive and therapeutic care, with the aim of improving quality of life and with pain and symptom relief. This is a literature review with bibliographic searches in scielo and pubmed databases with articles between 1993 and 2021. It was evident in this study that the dental surgeon plays a preponderant role in patient care and chemotherapy as these treatments can affect the oral cavity and together with a multidisciplinary team has the function of offering an improvement in the patient's quality of life and care before, during and even after anticancer treatment. It is necessary that the dental surgeon has adequate knowledge for the management and care of cancer patients.
\end{abstract}

Keywords: Head and neck neoplasms; Radiotherapy; Antineoplastic agents; Oncology; Dental care.

\section{Resumen}

El cáncer es una proliferación desordenada de células en tejido u órgano desencadenarse por factores externos e internos. El tratamiento antineoplásico divide en cirugía, radioterapia y quimioterapia, y puede usarse solo o en 
combinación. La radioterapia y la quimioterapia provocan efectos adversos en la cavidad oral del paciente, como xerostomía, mucositis oral, trismo, disfagia, odinofagia, candidosis, caries relacionada con la radiación y osteorradionecrosis. Necesario que el cirujano dental sepa diseñar el mejor protocolo de prevención, diagnóstico y tratamiento de forma individual para obtener los resultados deseados. Ojetivo de este trabajo es mostrar la importancia del cirujano dentista en el equipo multidisciplinar y su papel es fundamental en el seguimiento antes, durante y después de que el paciente se encuentre en tratamiento antineoplásico, brindándole cuidados preventivos y terapéuticos, con el objetivo de mejorar la calidad. de vida y con alivio del dolor y los síntomas. Se trata de una revisión de la literatura con búsquedas bibliográficas en bases de datos scielo y pubmed con artículos entre 1993 y 2021. En este estudio se evidenció que el cirujano dentista juega un papel preponderante en la atención al paciente y la quimioterapia ya que estos tratamientos pueden afectar la cavidad bucal y junto con un equipo multidisciplinar tiene la función de ofrecer una mejora en la calidad de vida y cuidados del paciente antes, durante e incluso después del tratamiento contra el cáncer. Es necesario que el cirujano dental tenga los conocimientos adecuados para el manejo y cuidado de los pacientes con cáncer.

Palabras clave: Neoplasias de cabeza y cuello; Radioterapia; Antineoplásicos; Oncologia; Atención odontológica.

\section{Introdução}

Neoplasia é o nome usado para definir um grupo de doenças que se caracteriza pela multiplicação desordenada das células de um tecido ou órgão, dentre esse grupo há uma divisão dessas desordens que são denominadas benignas ou malignas. As malignas são chamadas de câncer, podendo ser oriundo de fatores externos que são associadas ao ambiente, hábitos deletérios como o uso de álcool, tabaco e internos que estão relacionados a genética (Oppermann et al., 2014). As benignas têm células mais semelhantes à sua origem, velocidade de crescimento tumoral e a invasão local é menor e ficam restritas a uma cápsula fibrosa que impede a propagação desordenada desse amontoado de células defeituosas e não causando metástase (Medrado, 2015). Já as malignas as células não apresentam tanta semelhança com a sua célula de origem e se multiplicam de forma desordenada, podendo migrar para outros órgãos e tecidos causando metástase, podendo ser muito invasivo (Mitchell, Kumar, Abbas, \& Aster, 2017). Tanto as benignas quanto as malignas trazem complicações para o paciente, podendo ser mínimas como um problema na estética, ou levar a óbito. Assim, todos os diagnosticados com tumores devem ser acompanhados e submetidos a cuidados e tratamentos (Oppermann et al., 2014).

Algumas neoplasias podem dificultar um pouco o diagnóstico, entretanto as características que são mais avaliadas são diferenciação celular e anaplasia, velocidade de crescimento tumoral, invasão local e metástase. Para o tratamento do câncer existe uma gama de tratamentos antineoplásicos, a definição de qual será aplicado no paciente será conforme o tipo, localização, desenvolvimento entre outros fatores que são determinantes. Os tratamentos são divididos em remoção cirúrgica, radioterapia e quimioterapia ou associação de ambas (Medrado, 2015).

Pacientes que são submetidos a terapias antineoplásicas apresentam complicações durante e mesmo após o tratamento, algumas ocorrem na área da região de cabeça e pescoço que é a área de atuação do cirurgião dentista. Entre essas complicações estão mucosite, trismo, xerostomia ou hipossalivação, dificuldade de deglutição, alteração e perda do paladar, lesão de cárie dental causada pela radiação, osteorradionecrose, infecções oportunistas, problemas estéticos entre outros, que necessitam de cuidados preventivos e terapêuticos (Neville et al., 2016).

Neste trabalho mostraremos a importância do cirurgião dentista na equipe multidisciplinar e que seu papel é fundamental no acompanhamento antes, durante e mesmo após o paciente ser submetido ao tratamento antineoplásico, proporcionando cuidados preventivos e terapêuticos, com o intuito da melhora da qualidade de vida e com alívio da dor e sintomas.

\section{Metodologia}

O presente trabalho consistiu em uma análise descritiva qualitativa desenvolvida através de uma revisão de literatura. Os materiais foram obtidos por meio de livros e de artigos buscando as palavras chaves neoplasias de cabeça e pescoço, 
radioterapia, antineoplásicos, oncologia e cuidados odontológicos, utilizando os filtros temporais o período de 1993-2021 e filtros espaciais as bases de dados Scielo e Pubmed, com a intenção de analisar, ler e trazer os tópicos mais importantes sobre o assunto, através de trabalhos e pesquisas já realizadas.

\section{Revisão de Literatura}

\subsection{Entendendo o câncer}

O câncer é uma doença conhecida desde os primórdios da humanidade, há dados que mostram sua existência desde 1500 antes de Cristo, existe um papiro do Egito antigo que descreve alguns casos de câncer de mama, considerado na época sem cura, tornando possível somente o tratamento paliativo, visando promover a qualidade de vida do paciente. Atualmente temos acesso a tratamentos para o câncer que se dividem em quimioterapia, radioterapia, remoção cirúrgica e também tratamentos paliativos (Oppermann et al., 2014).

Câncer é a proliferação desordenada de células de um tecido ou órgão diferente da multiplicação que ocorre nas células normais que se divide em nascimento, amadurecimento e morte, as células neoplásicas continuam crescendo incontrolavelmente e formando novas células com as mesmas alterações, podendo haver uma proliferação rápida extremamente agressiva, trazendo um acúmulo dessas células vindo a formar uma massa que recebe o nome de tumor ou neoplasia (Medrado, 2015; Oppermann et al., 2014).

Essa multiplicação desordenada é desencadeada por alguns fatores, os internos que se trata da genética e externos que incluem vários fatores como uso de álcool e tabaco, exposição solar, alimentação, entre outros (Galbiatti, 2013).

O diagnóstico ocorre conforme as características, sintomas, utiliza-se imagens radiográficas, endoscópicas entre outros. Para fechar o diagnóstico é necessário a realização da biópsia que é analisada por um patologista, através dos dados obtidos é traçado pelo médico oncologista um tratamento com o paciente podendo ser ele radioterápico, quimioterápico, remoção cirúrgica, usados em conjunto ou não (Kignel, 2020; Oppermann et al., 2014).

\subsection{Tratamentos antineoplásicos}

\subsubsection{Radioterapia}

A radioterapia é o uso controlado de radiação para fins terapêuticos, ela é indicada com inúmeras finalidades, radioterapia curativa, pré-operatória, pós-operatória ou quimioterápica, paliativa (Medrado, 2015). A radiação ionizante é usada para causar a morte celular, principalmente quando se trata de neoplasias malignas. Ela surgiu no início do século XX, antes do uso dos quimioterápicos e era associada a cirurgia (Camargo, 2015; Eduardo, Bezenelle, Corrêa, Waksman, \& Farah, 2019).

A radioterapia é o tratamento mais usado com intuito curativo ou paliativo em caso de neoplasias sólidas (Cobos et al., 2019). Ela utiliza equipamentos e técnicas para irradiar áreas específicas com objetivo de impedir a multiplicação das células neoplásicas de forma local. Atualmente já é usado técnicas de alta precisão fazendo com que o tratamento se torne mais eficaz e trazendo mais segurança para o paciente (Camargo, 2015; Medrado, 2015).

A radiação ionizante é dividida em três categorias, radiação alfa, beta e gama. Elas agem nas células tumorais e o DNA é o principal ponto de ação, ela causa danos ao DNA, mas também pode agir de forma indireta interagindo com os átomos e moléculas e em especial na água, ganhando o nome de radiólise da água, produzindo radicais livres com a capacidade de desencadear radiações químicas com diferentes alvos (Cobos et al., 2019).

A radioterapia apresenta efeitos tóxicos que variam conforme a neoplasia, a energia e volume do tecido irradiado, a saúde do paciente também é um fator importante, há reações adversas muito comuns e acontecem em pacientes que são 
submetidos ao tratamento independente dos fatores citados. Os efeitos são divididos em dois grupos se tratando dos efeitos imediatos que são mais comuns e os efeitos tardios que são mais raros (Camargo, 2015).

\subsubsection{Quimioterapia}

A quimioterapia é basicamente um tratamento à base de quimioterápicos ou antineoplásicos que são induzidos no corpo do paciente com objetivo de atingir as células cancerígenas presente no organismo (Medrado, 2015).

O tratamento quimioterápico segue uma cronologia, sendo essa a seguinte: quimioterapia por indução é a terapia inicial, quimioterapia de consolidação é feita depois da remissão da doença, quimioterapia adjuvante utilizada depois da erradicação da doença com tratamento local, quimioterapia neoadjuvante é usada antes da terapia local, quimioterapia de manutenção onde é feito a administração prolongada com baixas doses e tem o objetivo de prolongar a remissão e atingir a cura e a quimioterapia de resgate é utilizada posteriormente ao fracasso dos outros tratamentos (Govindan \& Arquette, 2014).

A aplicação dos fármacos pode ser administrada por via oral, por injeção intravenosa, intramuscular, subcutânea, por via intracraneal, intra abdominal e uso tópico podendo ser feita a utilização de um ou mais fármacos (Barrios, Sampaio, \& Vasconcelos, 2013; Medrado, 2015). Após a sua aplicação o quimioterápico cai na corrente sanguínea e se espalha pelo corpo, isso independe da forma que é feita a sua indução no organismo, a mesma não influencia no seu efeito (Barrios et al., 2013).

Os quimioterápicos recebem uma divisão de acordo com sua atuação no ciclo celular, os quimioterápicos de ciclo não específicos que agem nas células que estão ou não se proliferando e os de ciclos específicos que agem somente nas células que estão em proliferação, mas eles também podem ser classificados de acordo com seu mecanismo de ação podendo ser eles antimetabólicos, alquilantes e inibidores mitóticos (Rodrigues \& Oliveira, 2016).

A droga aplicada, o tempo de exposição e a concentração interferem na resposta do organismo. Os fármacos quimioterápicos não atuam somente nas células antineoplásicas, eles podem apresentar efeitos em células saudáveis como a medula óssea, o tubo digestivo e nos pêlos presentes no corpo como por exemplo, no cabelo, entretanto as células normais apresentam uma recuperação previsível e as células neoplásicas não, por isso há uma necessidade de tempo para aplicação dos quimioterápicos para que haja a recuperação das células saudáveis (Medrado, 2015).

\subsubsection{Cirurgia}

As cirurgias são o tratamento mais antigo utilizado contra o câncer, o objetivo principal é a remoção da massa cancerígena para assim amenizar sua proliferação (Medrado, 2015). A cirurgia traz inúmeros benefícios ao paciente submetido a esse tratamento, não causa tantos malefícios ao organismo quanto a quimioterapia e a radioterapia, mas pode trazer risco de morte durante o procedimento, deformação e perda de função, e não é possível ser utilizada em todos os casos (Lopes, Rossi, \& Nakagawa, 1993; Medrado, 2015).

As modalidades cirúrgicas são divididas em categorias, a biópsia que é utilizada para o diagnóstico da doença, a cirurgia curativa usada em estágios iniciais da doença que se trata da remoção das áreas neoplásicas, cirurgias paliativas para diminuir a dor do paciente e oferecer mais qualidade de vida. Para decidir a modalidade a ser usada deve ser estudado o caso de cada paciente (Barrios et al., 2013; Medrado, 2015).

\subsubsection{Cuidados paliativos}

Os cuidados paliativos (CP) são os cuidados realizados no paciente no final da vida, se torna necessário uma equipe multidisciplinar composta por médicos, enfermeiros, psicólogos, assistentes sociais, nutricionistas, dentistas, fisioterapeuta, fonoaudiólogos entre outros profissionais. O seu papel é oferecer uma melhora na qualidade de vida do paciente e de sua 
família, um dos maiores objetivos é conduzir de forma adequada a melhora da dor e dos sintomas para garantir uma qualidade de vida durante sua terminalidade (Rodrigues, 2018).

A Organização Mundial da Saúde (OMS) descreve que o cuidado paliativo deve ocorrer de forma continua a partir do diagnóstico e paralelo ao tratamento que busca a cura ou controle da doença, muitas vezes com a evolução da doença torna-se necessário o aumento desse cuidado por talvez se tornar a única forma terapêutica possível durante o processo de morte (Velasco \& Ribeiro, 2021).

\subsection{Complicações odontológicas no tratamento antineoplásico}

Atualmente todos os tratamentos antineoplásicos existentes são agressivos e causam danos biológicos, tornando o sistema estomatognático extremamente frágil, não atingindo somente as células neoplásicas, afetando também as células saudáveis, principalmente os tecidos de renovação rápida como por exemplo, o epitélio da cavidade oral, resultando em complicações graves (Hespanhol, Tinoco, Teixeira, Falabella, \& Assis, 2010).

Se tratando de tratamentos antineoplásicos quanto mais potente mais danos são causados a cavidade oral, tanto a radioterapia como a quimioterapia podem trazer efeitos colaterais. Os efeitos podem ser agudos ou crônicos, todos eles afetam a qualidade de vida do paciente e na efetividade do tratamento oncológico podendo causar inclusive a interrupção do mesmo (Ghelardi et al., 2008).

As reações agudas acontecem durante o tratamento e em sua grande maioria são reversíveis, já as complicações crônicas podem surgir após o final do tratamento em geral, são irreversíveis e sendo dividas em leves, moderadas e graves (Jham, \& Freire, 2006). As manifestações orais dependem do campo irradiado, da dose e da idade do paciente, as consequências possíveis são divididas em agudas que são mucosite, alteração do paladar, trismo, candidose e xerostomia, e as crônicas que são osteorradionecrose, cárie por radiação e também xerostomia (Marcucci, \& Junior, 2020).

\subsubsection{Xerostomia nos tratamentos antineoplásicos}

A xerostomia se trata da sensação de boca seca, podendo ou não haver a queda da quantidade do fluxo salivar, quando isto ocorre acontece alterações de muitos fatores como por exemplo, a queda das funções microbianas, bioquímicas e fisiológicas da saliva na cavidade bucal (Marcucci, \& Junior, 2020).

As glândulas salivares apresentam grande fragilidade quando expostas à radioterapia, e por esse motivo a xerostomia é a manifestação oral que mais acomete pacientes submetidos a esse recurso terapêutico, essas alterações surgem normalmente a partir da primeira semana de tratamento com a diminuição do fluxo salivar que pode ser notada durante às seis primeiras semanas de tratamento (Galbiatti, 2013).

A xerostomia também pode ser causada pelo tratamento quimioterápico, a droga antineoplásica que causa xerostomia com mais frequência é a doxorrubicina, pesquisas mostram que 100\% dos pacientes com fluxo salivar reduzido anteriormente a terapia com quimioterápicos vem apresentar xerostomia independente do quimioterápico, por isso a importância de medir o fluxo salivar previamente (Martins, Caçador, \& Gaeti, 2002).

A hipossialia é outro termo usado para descrever a xerostomia, para identificarmos se o paciente apresenta essa alteração o seu fluxo salivar não estimulado deve ser menor que $0,1 \mathrm{ml}$ por minuto, e deve ser medido durante 5 a 15 minutos, ou quando o fluxo salivar estimulado for menor que $0,7 \mathrm{ml}$ por minuto e ser medido ao longo de 5 minutos (Marcucci, \& Junior, 2020).

Os pacientes que apresentam xerostomia provenientes de tratamento antineoplásico desenvolvem inúmeras consequências da diminuição salivar, como por exemplo, dificuldades durante a alimentação, fala, ao dormir, afeta também o uso de próteses e aumenta as chances do desenvolvimento de cáries (Galbiatti, 2013). 
Cuidados e tratamentos relacionados a xerostomia

O tratamento da xerostomia proveniente dos recursos terapêuticos antineoplásicos é o mesmo de quando essa alteração é resultante de outros fatores. Deve se recomendar ao paciente que não faça o uso de álcool e tabaco, reforçar a hidratação com o aumento da ingestão de água, sendo possível também a realização da estimulação mecânica e ingestão de fármacos como por exemplo, a pilocarpina e a cevemelina (Eduardo et al., 2019; Marcucci, \& Junior, 2020; Neville et al., 2016).

A saliva artificial também é um tratamento muito usado para auxiliar no controle da xerostomia, minimizando assim, o desconforto e a sensação de boca seca trazendo a melhora na qualidade de vida do paciente. Ela é indicada com frequência para pacientes submetidos a tratamentos oncológicos (Eduardo et al., 2019).

Um cuidado que deve sempre ser tomado é em relação às cáries causadas pela xerostomia, a saliva é um fator importante na proteção na cavidade oral, quando ocorre a diminuição da saliva os dentes ficam mais propensos a desenvolver cárie, a aplicação diária de flúor tópico (fluoreto de sódio neutro 1\%) ajuda a prevenir o surgimento da cárie devendo ser associada aos cuidados citados anteriormente (Neville et al., 2016).

\subsubsection{Mucosite oral nos tratamentos antineoplásicos}

A mucosite é considerada uma das manifestações orais mais debilitantes provenientes de tratamentos antineoplásicos, $80 \%$ dos pacientes submetidos a radioterapia na região da cabeça e pescoço apresentam essa complicação, tratando-se de radioterapia de orofaringe e boca essa porcentagem sobe para 100\%, já quando associada a quimioterapia sua ocorrência se altera segundo o protocolo adotado (Neville et al., 2016).

Em muitos casos a mucosite pode trazer a necessidade de internação hospitalar por longos prazos, aumentando os custos e qualidade do tratamento, às vezes, se tornando necessário o uso de nutrição parenteral no paciente e até mesmo a interrupção do tratamento até a melhora do quadro clínico (Carvalho, Souza, Franck, Polastrini, \& Crispim, 2018).

Tanto a mucosite causada pela radioterapia, quanto pela quimioterapia apresentam características clínicas semelhantes, as manifestações quando associadas a quimioterapia surgem em alguns dias, quando se trata da radioterapia elas aparecem na segunda semana de tratamento, é caracterizada por uma lesão eritematosa, apresentando edema, ulceração e recoberta por uma pseudomembrana com halo eritematoso, podendo ser acompanhada de sangramento e causar dor intensa (Carvalho et al., 2018; Neville et al., 2016).

\section{Cuidados e tratamentos relacionados a mucosite}

Alguns tratamentos são utilizados com propósito de prevenir o surgimento da mucosite oral, como por exemplo, a crioterapia que é a colocação de raspas de gelo na cavidade oral 5 minutos antes do tratamento por 30 minutos e tem demonstrado efetividade quando se trata de quimioterápicos com meia vida curta. A crioterapia não apresenta efeitos quando associada a radioterapia, nesse tratamento é necessário a utilização de outros mecanismos como por exemplo, o uso de bloqueadores de radiação na linha média ou uso de radiação tridimensional para limitar o volume da mucosa irradiada (Neville et al., 2016).

Atualmente há uma grande utilização da laserterapia de baixa potência em pacientes com mucosite para a melhora da qualidade de vida, o laser atua na cavidade bucal como anti-inflamatório, analgésico, aumenta o metabolismo da célula ajudando assim na sua cicatrização e também no tratamento e prevenção da mucosite oral oferecendo uma maior integridade para cavidade oral e uma melhor qualidade de vida (Spezzia, 2015).

Além de tudo que foi citado anteriormente deve ser oferecido ao paciente as instruções de higiene oral e apoio nutricional. Atualmente todos os tratamentos relacionados a mucosite oral são apenas paliativos e pode também ser feito 
através de terapias com uso de fármacos, como fatores de crescimento, vitamina E, antimicrobianos, anti-inflamatórios, enxaguatórios bucais, anestésicos tópicos e antioxidantes (Sousa, 2011).

\subsubsection{Trismo nos tratamentos antineoplásicos}

O trismo é uma alteração que pode surgir em cerca de $45 \%$ dos pacientes que são submetidos a radioterapia em doses curativas na região de cabeça e pescoço. Trata-se de uma contração tônica dos músculos da mastigação que como resultado tem a limitação da abertura bucal, podendo surgir nos primeiros 3 dias de RT (radioterapia), por muitas vezes o paciente nota desde que ela está associada a alguma dor ou disfunção que podem vir a afetar o estado nutricional, habilidade de higienização e fonação, apresentando futuras complicações no tratamento e o diagnóstico é feito clinicamente (Carvalho et al., 2018).

\section{Cuidados e tratamentos relacionados ao trismo}

Os cuidados relacionados devem ser durante a fase de planejamento da RT de cabeça e pescoço, com a intenção de minimizar o máximo possível a radiação no aparelho mastigatório, outra habilidade que também é usada como prevenção antes do trismo se estabelecer são exercícios de alongamento da mandíbula. Quando já estabelecido torna-se muitas vezes necessário uma equipe multidisciplinar para o tratamento que é composta por médicos, nutricionista, cirurgião dentista, fisioterapeuta e fonoaudiólogo. Dentre os métodos terapêuticos que podem ser oferecidos pelo cirurgião dentista estão a cinesioterapia, ultrassom terapêutico, neuroestimulação elétrica transcutânea e laserterapia de baixa potência (Carvalho et al., 2018; Melo et al., 2015).

\subsubsection{Disfagia e odinofagia nos tratamentos antineoplásicos}

A disfagia é descrita como dificuldade para engolir e a odinofagia é a dor ao engolir, elas são efeitos colaterais que podem surgir durante a terapia antineoplásica. Pacientes com disfagia e odinofagia podem apresentar perdas nutricionais que afetam diretamente no tratamento, além disso, 1/3 podem desenvolver pneumonia. A ocorrência e intensidade é segundo o protocolo utilizado, deve ser salientado ao paciente que ele pode desenvolver ou piorar a disfagia mesmo após o fim do tratamento e existem alguns fatores de riscos que podem aumentar as oportunidades do paciente apresentar a disfagia e odinofagia que são quimioterápicos agressivos, xerostomia e tabagismo ativo (Marcucci, \& Junior, 2020).

\section{Cuidados e tratamentos relacionados a disfagia e odinofagia}

Os cuidados relacionados a disfagia e odinofagia começam antes da realização dos tratamentos antineoplásicos, a capacidade do paciente de suportar dor e doses mais altas de opióides é um fator importante a ser considerado, pessoas mais frágeis como idosos podem apresentar intolerância aos medicamentos utilizados para lidar com a dor oral associadas a uma terapia mais agressiva (Marcucci, \& Junior, 2020).

O tratamento é feito com administração de analgésicos proporcionalmente a dor, com fim de aliviá-la, sendo de grande importância o esforço para manter a deglutição durante e após o tratamento antineoplásico com a finalidade de diminuir a atrofia por desuso e aperfeiçoamento da função a longo prazo (Marcucci, \& Junior, 2020).

\subsubsection{Candidose nos tratamentos antineoplásicos}

Pacientes que são submetidos a tratamentos antineoplásicos podem vir a desenvolver a candidose, que se trata de uma infeção causada por um fungo que em sua grande maioria é a candida albicans, podendo surgir tanto durante como após a terapia, podendo apresentar sintomas como dor, disgeusia, anorexia e desnutrição. Fatores como lesões na mucosa, uso de próteses sem higienização adequada, tabagismo e/ou etilismo aumentam o risco de candidose, e a hipossalivação eleva o risco 
de colonização e infecção. As formas clínicas da candidose são descritas como pseudomembranosa, eritematosa, queilite angular, hiperplásica crônica e mucocutânea (Govindan \& Arquette, 2014; Marcucci, \& Junior, 2020; Neville et al., 2016).

\section{Cuidados e tratamentos relacionados a candidose}

Os fatores de risco para o surgimento da candidose devem ser identificados e eliminados sempre que possível antes do surgimento da doença, deve-se instruir o paciente em relação aos cuidados orais em relação à higiene bucal e eliminação de hábitos deletérios como tabagismo e/ou etilismo para a prevenção. O tratamento da candidose é dividido em tópico e sistêmico, sendo esses agentes poliênicos (nistatina e anfotericina B), agentes imidazólicos (clotrizamol e cetoconozol), triazóis (fluconazol itraconazol, posaconozol equinocandinas), sempre que possível optar pelo antifúngico tópico por ser menos invasivo, os sistêmicos devem ser opção de escolha quando somente o tópico não for suficiente e em casos mais graves são usados os dois em conjunto (Marcucci, \& Junior, 2020; Neville et al., 2016).

\subsubsection{Cáries induzidas por radiação}

Os principais fatores etiológicos da cárie por radiação são alterações que ocorrem na saliva tanto qualitativas como quantitativas devido a danos causados pela radioterapia nas glândulas salivares que trazem variações imunológicas e químicas na saliva, como por exemplo, a hipossalivação e modificação do pH da saliva. Também ocorre mudanças na superfície de dentes irradiados onde há diminuição da vascularização da polpa, variações da função da síntese dos odontoblastos e colágeno, assim ocorrendo a diminuição do potencial reparativo da polpa (Eduardo et al., 2019).

Clinicamente a cárie por radiação tem início nas superfícies vestibulares cervicais dos dentes e atingem também as lisas, inclusive de dentes anteriores e inferiores, o que é anormal em outras situações vistos que esses dentes apresentam maior resistência a cárie em outras circunstâncias, mas a predileção pela região anterior acontece, pois, a área é mais afetada pela diminuição do fluxo salivar. Ocorrem mudanças também na translucidez e na cor da estrutura dentária que inicialmente apresenta coloração esbranquiçada e posteriormente ocorre a evolução para negra-acastanhada, aumentando a friabilidade e a degradação da estrutura dentária podendo acarretar a amputação completa da coroa (Eduardo et al., 2019; Marcucci, \& Junior, 2020).

Existem três categorias de cárie por radiação, a tipo 01 é a que apresenta maior incidência, a sua extensão vai até a junção cemento-esmalte, ocorre uma deterioração circunferencial e consequentemente a constrição dessa região que normalmente é a região cervical e por esse motivo pode apresentar com grande frequência a amputação da coroa, tipo 02 ela surge como áreas de desmineralização em toda a superfície dental e desenvolve erosões de forma generalizada e a oclusal e incisal desgastadas são visíveis e a tipo 03 que é a mais incomum é vista uma mudança de coloração na dentina e a coroa se torna marrom escura e acontece um desgaste na incisal e oclusal (Emerim et al., 2018).

\section{Cuidados e tratamentos relacionados a cárie por radiação}

Os cuidados relacionados a cárie começam antes da radioterapia na região de cabeça e pescoço onde deve ser passado aos pacientes os cuidados em relação à higiene oral que precisam ser mantidos durante e após o tratamento, visto que as cáries por radiação podem surgir mesmo após o tratamento finalizado. A necessidade da realização da adequação da cavidade bucal e remoção dos possíveis focos infecciosos, são também necessários cuidados que previnam o surgimento da xerostomia. Durante o protocolo radioterápico é recomendado aplicações tópicas de flúor feitas através de bochechos diários com soluções contendo fluoreto de sódio 0,5 a 1\%, uso de dentifrícios com suplementação de cálcio e fosfato (Eduardo et al., 2019).

Quando ocorre o surgimento da lesão cariosa, a restauração pode apresentar um desafio devido a mudanças que ocorrem na estrutura dentária e podem afetar de forma direta a efetividade do processo, o recomendado seria a remoção das 
lesões cariosas previamente ao tratamento radioterápico sempre que possível e quando não houver possibilidade deve-se realizar restaurações com ionômero de vidro e após o fim da radioterapia a realização das restaurações definitivas (Eduardo et al., 2019; Marcucci, \& Junior, 2020).

\subsubsection{Osteorradionecrose associada a radioterapia}

A osteorradionecrose é uma das consequências mais graves proveniente do tratamento radioterápico na região de cabeça e pescoço, é definida como exposição do osso previamente irradiado, que falha na reparação por um período maior que 3 meses, pressupõe-se que ele apresente hipocelularidade, hipovascularização e hipóxia resultando na incapacidade correta de reparação, há uma diminuição na atividade osteoblástica e osteoclástica e a reparação ocorre por cicatrização causando a formação de um tecido fibroso (Eduardo et al., 2019; Neville et al., 2016).

Podendo ser tanto assintomática como sintomática, pacientes edêntulos apresentam menos risco de desenvolver osteorradionecrose, sendo mais comum seu surgimento na área da mandíbula visto que apresenta uma irrigação menor que a maxila, sua prevalência varia de 5 a $15 \%$ e acomete com maior frequência pacientes com mais de 55 anos. Alguns fatores podem aumentar o risco do seu surgimento como por exemplo, a dose de radiação, modalidade de tratamento, o fracionamento da radioterapia, higiene oral, exodontias e outros procedimentos cirúrgicos após o tratamento, tabagismo e/ou etilismo (Eduardo et al., 2019; Marcucci, \& Junior, 2020).

\section{Cuidados e tratamentos relacionados a osteorradionecrose}

Entre os cuidados relacionados a osteorradionecrose o principal é a prevenção. Deve ser inserida uma ótima higiene oral e mantida durante e após o tratamento, é necessária a adequação do meio bucal antes do início do tratamento radioterápico onde deve ser extraído todos os dentes que não apresentam probabilidade de tratamento restaurador e é necessário um intervalo de 7 a 14 dias após a realização de procedimentos invasivos e o início do tratamento radioterápico para possibilitar a cicatrização dos tecidos, alguns autores recomendam que seja aguardado até 21 dias, e é de suma importância que a técnica cirúrgica seja a mais atraumática possível (Neville et al., 2016).

Durante o tratamento não é recomendado a realização de exodontias, quando se trata de extrações realizadas após a radioterapia são necessários estudos adicionais para determinar o prazo exato entre a realização da extração e o fim do tratamento radioterápico para determinar se há ou não o risco de desenvolver osteorradionecrose. O período de 2 a 5 anos após a radioterapia apresentou mais incidência de osteorradionecrose quando realizado extrações, no estudo realizado por Bonan em 2006, houve uma média de 2,3 meses entre o fim da radioterapia e início da osteorradionecrose, podendo variar de 1 a 10 meses (Emerim et al., 2018; Gupta et al., 2015).

Em relação ao tratamento é recomendado antibioticoterapia preventiva antes do surgimento da osteorradionecrose, e quando ela já está estabelecida a antibioticoterapia é realizada em altas dosagens, acompanhada com irrigação com soluções salinas ou clorexidina $0,12 \%$, analgésicos como tramadol e morfina para alívio da dor, anti-inflamatório não esteroidais podem ser prescritos. Bem como a utilização de ultrassom de ondas curtas para produzir neovascularização e neoformação celular que apresentam efetividade em alguns casos (Emerim et al., 2018; Marcucci, \& Junior, 2020; Pozzobon et al., 2011).

Pode ser utilizado o laser de baixa intensidade para o auxílio do tratamento, também é recomendado a realização da remoção dos tecidos necrosados com a intenção da cicatrização por primeira intenção e em alguns casos a cirurgia ablativa de tecidos moles e duros. A oxigenação hiperbárica é um procedimento usado como terapia e consiste na inalação de oxigênio puro através de uma pressão atmosférica aumentada, podendo estar associado ou não a cirurgia. Os pacientes com osteorradionecrose devem ser acompanhados e realizado exames com imagens a cada 6 meses ou menos (Emerim et al., 2018; Marcucci, \& Junior, 2020; Pozzobon et al., 2011). 


\subsubsection{Cuidados odontológicos gerais antes, durante e após o tratamento antineoplásico}

Os cuidados odontológicos prévios aos tratamentos antineoplásicos têm como papel a eliminação de oportunidades de futuras complicações orais que podem interferir de forma negativa na oncoterapia. $\mathrm{O}$ cirurgião dentista deve fazer um plano de tratamento inicial, deve se levar sempre em consideração a colaboração do paciente, dentes que em outras situações teriam um prognóstico melhor devido ao caráter de urgência em alguns casos devem ser extraídos para que seja evitado complicações como as descritas anteriormente (Marcucci, \& Junior, 2020; Santos, 2016).

Entre os procedimentos curativos que devem ser realizados previamente ao tratamento estão o ajuste das próteses removíveis e fixas, tratamento das lesões estomatognáticas dos tecidos mineralizados e moles, remoção de todos os focos de infecção, os dentes com um prognóstico não tão favorável devem ser extraídos, em geral, deve ser feito a adequação do meio bucal (Marcucci, \& Junior, 2020).

Quando se fala em tratamento preventivo alguns cuidados são importantes e devem ser seguidos como identificar o risco, o paciente deve ser instruído em relação aos cuidados de higiene oral que deve ser realizada de 02 a 04 vezes ao dia com uma escova de cerdas macias e o fio dental deve ser usado diariamente, caso o paciente faça o uso de próteses deve ser feita a higienização das mesmas, também é recomendado a utilização de enxaguantes bucais como por exemplo, a clorexidina $0,12 \%$, existe a necessidade do abandono de hábitos deletérios como alcoolismo, tabagismo e o paciente deve estar sempre sob acompanhamento odontológico (Marcucci, \& Junior, 2020). A profilaxia com flúor deve ser realizada no paciente, é recomendado o uso de substitutos salivares em alguns casos para diminuir os problemas causados pela diminuição da saliva, quando há dor é indicado o uso de analgésico tópico e sistêmico e se necessário uso de agente anestésico sistêmico. Caso o paciente apresente mucosite o laser de baixa potência apresenta grande eficiência no alívio dos sintomas e também pode ser utilizado para prevenção. Extrações dentárias durante o tratamento não são indicadas visto que podem causar complicações como osteorradionecrose e deve ser evitada a realização deste procedimento (Pozzobon et al., 2011; Santos, 2016).

O paciente submetido a oncoterapia necessita de acompanhamento odontológico após o final do tratamento, pois pode ocorrer o surgimento de efeitos odontológicos tardios que devem ser tratados, lesões de cárie devem ser removidas e o dente restaurado e extrações dentárias precisam ser evitadas sempre que possível. É importante que os cuidados relacionados a higiene oral seja mantido mesmo com o tratamento antineoplásico finalizado. É indicado uso de flúor em gel e clorexidina para o paciente de forma contínua com a intenção da redução da incidência de cáries relacionadas à radiação (Pozzobon et al., 2011; Spezzia, 2015).

\section{Discussão}

Segundo a Organização Mundial da Saúde (OMS) e o Instituto Nacional do Câncer (INCA) nas próximas décadas haverá um aumento significativo do número de pacientes acometidos com o câncer, sendo de suma importância conhecer melhor a doença, os tratamentos e as complicações que podem surgir para poder conduzir da melhor forma e saber os cuidados que devem ser tomados frente aos efeitos colaterais na cavidade oral (Oppermann et al., 2014).

Os tratamentos oferecidos aos pacientes com câncer são divididos em radioterapia, quimioterapia e cirurgia, com objetivo da eliminação completa da doença, oferecer uma sobrevida prolongada ou aliviar o paciente das complicações advindas do câncer. Muitos dos pacientes em tratamento oncológico principalmente na região de cabeça e pescoço recebem uma destas modalidades terapêuticas ou associação de ambas que podem trazer efeitos adversos, sendo necessário tratamentos odontológicos preventivos e terapêuticos (Medrado, 2015)

Alguns protocolos são utilizados devido a algumas complicações estomatológicas dos tratamentos antineoplásicos, sendo necessário cuidados orais prévios, durante e após a oncoterapia para que seja diminuída ou evitada a incidência de problemas ocorrentes como xerostomia, mucosite oral, trismo, disfagia e odinofagia, candidose, cáries induzidas por radiação e 
osteorradionecrose, que podem causar perdas significativas de função, causando diminuição da qualidade de vida e até mesmo interrupção do tratamento até a melhora do quadro clínico (Jham \& Freire, 2006).

O dentista tem um papel fundamental no tratamento antineoplásico desde os diagnósticos até os cuidados paliativos, sendo responsável pela eliminação de diversos problemas e reconhecimento das lesões cancerígenas da cavidade oral. Devendo orientar os pacientes também em relação aos fatores externos que influenciam no surgimento do câncer como o uso do tabaco, com intenção da diminuição da incidência do câncer da cavidade oral (Castro et al., 2002).

Ao reconhecer as complicações orais decorrentes da terapia antineoplásica o dentista poderá atuar de forma efetiva na prevenção, tratamento e no monitoramento, oferecendo assim cuidados necessários que devem ser contínuos com intervalos regulares mantendo assim a integridade da cavidade oral e impedindo também o aparecimento de infecções secundárias (Gupta et al., 2015; Jham \& Freire, 2006).

\section{Conclusão}

É fundamental o papel do cirurgião dentista na equipe multidisciplinar e no atendimento odontológico dos pacientes oncológicos e que o acompanhamento seja realizado antes, durante e mesmo após o paciente ser submetido ao tratamento, a fim de prevenir, diagnosticar e tratar os efeitos colaterais e oferecer melhor qualidade de vida para os pacientes. Sendo importante que o profissional tenha o conhecimento necessário, para identificação do problema e saiba estabelecer um plano de tratamento eficiente de acordo com cada caso.

Novos trabalhos devem ser realizados para aprimorar ainda mais as possibilidades preventivas e terapêuticas nos cuidados odontológicos aos pacientes submetidos a tratamentos antineoplásicos.

\section{Referências}

Barrios, C. H. E., Sampaio, C. A. P., \& Vasconcelos, C. M. M. (2013). Tudo que você precisa saber sobre o câncer de mama. Minha editora.

Camargo, R. (2015). Radioterapia e medicina nuclear, conceitos instrumentação, protocolos, tipos de exame e tratamentos. Érica.

Carvalho, R. T., Souza, M. R. B., Franck, E. D., Polastrini, R. T. V., \& Crispim, D. (2018). Manual da residência de cuidados paliativos, abordagem multidisciplinar (1a. ed.). Barueri: Manole.

Castro, R. F. M., R., Dezotti, M. S. G. Azevedo, L. R., Aquilante, A. G., \& Xavier, C. R. G. (2002). Atenção odontológica aos pacientes oncológicos antes, durante e depois do tratamento antineoplásico. Rev odontol. UNICID, 14(1), 63-74.

Cobos, M. R., Ricardo, J. H., Molina, M. S., Plata, J. P., Lopez, L. C., Nieto, S. E.et al. (2019). Oral health status before, during and after antineoplastic treatment at a cancer institute in Barranquilla, Colombia. J Oral Res, 8(4), 316-324.

Eduardo, F. P., Bezenelle, L. M., Corrêa, L., Waksman, R. D., \& Farah, O. G. (2019). Odontologia Hospitalar. Manoele.

Emerim, J. S., Silveira, L. V. R., Lourenço, S. S., Braz, M. A., Brew, M. C., \& Bavaresco, C. S. (2018). O manejo das complicações bucais em pacientes sob tratamento oncológico na atenção básica. Revista saúde e ciência online, 7(3), 90-106.

Figuira, P., Marx, A., Figueira, P., \& Tacani, P. M. (2017). Manual de condutas e práticas de fisioterapia em oncologia, neoplasias de cabeça e pescoço (1a. ed.). Barueri: Manole.

Galbiatti, A. L. S., Padovani-Junior, J. A., Maníglia, J. V., Rodrigues, C. D. S., Pavarino, É. C., \& Goloni-Bertollo, E. M. (2013). Câncer de cabeça e pescoço: causas, prevenção e tratamento. Braz j otorhinolaryngol., 79(2),239-247

Ghelardi, I. R., Junior, C. A. V. S., Santos, P. S. S., Teixeira, S. S., Matsushita, C. M., Carrilo, C. M. et al. (2008). A necessidade da avaliação e tratamento odontológico pré-radioterapia. Prat. hosp. X(58), 149-151.

Govindan, R., \& Arquette, M. A. (2014). Washington manual de oncologia. Guanabara Koogan.

Gupta, N., Pal, M., Rawat, S, Grewal, M. S., Garg, H., Chauhan, D. et al. (2015). Radiation-induced dental caries, prevention and treatment - A systematic review. Natl J Maxillofac Surg., 6(2),160-166.

Hespanhol, F. L., Tinoco, E. M. B., Teixeira, H. G. C., Falabella, M. E. V., \& Assis, N. M. S. P. (2010). Manifestações bucais em pacientes submetidos à quimioterapia. Ciênc. saúde coletiva, 15(1), 1085-1094.

Jham, B. C., \& Freire, A. R. S. (2006). Complicações bucais da radioterapia em cabeça e pescoço. Rev Bras. Otorrinolaringol, 75(5),704-708. 
Research, Society and Development, v. 10, n. 12, e99101220231, 2021

(CC BY 4.0) | ISSN 2525-3409 | DOI: http://dx.doi.org/10.33448/rsd-v10i12.20231

Kignel, S. (2020). Estomatologia bases de diagnóstico para o clínico geral (3a ed.). Guanabara Koongan.

Lopes, A., Rossi, B. M, \& Nakagawa, W. (1993). Oncologia cirúrgica. Acta Oncol. Bras 113(1/3), 42-7.

Marcucci, G., \& Junior, O. C. (2020). Fundamentos de odontologia: estomatologia. (3a ed.). Guanabara Koogan.

Martins, A. C. M., Caçador, N. P., \& Gaeti, W. P. (2002). Complicações bucais da quimioterapia antineoplásica. Acta sci., 24, 663-670.

Medrado, L. (2015). Carcinogênese desenvolvimento, diagnóstico e tratamento das neoplasias. Erica.

Melo, A. U. C. M., Ribeiro, C. F., Sobrinho, J. R. P., Calasans, F. J. C. P., Nascimento, M. J., \& Carvalho, F. L. O. (2015). Trismo decorrente da radioterapia em cabeça e pescoço - abordagem fisioterápica. Clipe Odonto, 7(1), 37-44.

Mitchell, R. N., Kumar, V., Abbas, A. K., \& Aster, J. C. (2017). Robbis e Contran fundamentos de patologia (9a. ed.). Elsevier.

Neville, B. W., Damm, D. D., Allen, C. M., \& Chi, A. C. (2016). Patologia oral e maxilofacial (4a ed.). Elsiever2016.

Oppermann, C. P., Azambuja, A. A., Fischer, C. A. C., Barrios, C. H., Prolla, G., Geib, G. et al. (2014). Entendendo o câncer (61a. ed.). Artmed.

Pozzobon, J. L., Ortiz, F. R., Braun, K., \& Unfer, B. (2011). Complicações bucais dos tratamentos de câncer de cabeça e pescoço e de malignidades hematológicas. $R F O, 16(3), 342-346$.

Rodrigues, A. B., \& Oliveira, P. P. (2016). Oncologia para enfermagem. Manole.

Rodrigues, K. M. (2018). Princípios dos cuidados paliativos. SAGAH.

Santos, L. C. (2016). O papel do Cirurgião Dentista na equipe multidisciplinar de oncologia. Trabalho de Conclusão de Curso de Bacharelado em Odontologia, Centro Universitário do Planalto Central Apparecido dos Santos.

Sousa, R. C. (2011). Interfaces entre radioterapia e odontologia. Monografia de Especialização em Radiologia e Imaginologia, Universidade Federal do Rio Grande do Sul., Porto Alegre.

Spezzia, S. (2015). Mucosite oral. J Oral Invest., 4(1), 14-18.

Velasco, I. T., \& Ribeiro, S. C. C. R. (2021). Cuidados paliativos na emergência. Manole. 